\title{
Efectos de una simetría facial alta, media y baja en un condicionamiento clásico de actitudes hacia logos*
}

\author{
Effects of high, medium and low facial symmetry \\ in a classical conditioning of attitudes of logos
}

\author{
Danilo Zambrano R." \\ Julián David Agudelo T. \\ Universidad de Ibagué, Colombia \\ Lady Grey Javela \\ Universidad del Rosario, Colombia \\ Julio Eduardo Cruz \\ Universidad de los Andes, Colombia \\ Recibido: 2 de diciembre de 2015 \\ Revisado: 3 de enero de 2016 \\ Aceptado: 30 de enero de 2016
}

\section{Resumen}

Con 34 estudiantes de la Universidad de Ibagué (17 de cada sexo y una media de 21 años), se evaluó el efecto de la simetría facial (SF) en un Condicionamiento Clásico de Actitudes, mediante un procedimiento simultáneo donde los estímulos incondicionados fueron rostros femeninos con baja, media y alta SF, y como estímulos a condicionar tres logos (Xua, Xue y Xuo). Los resultados muestran una mejor evaluación de los rostros con alta SF; un cambio entre las evaluaciones realizadas antes y después a los logos $y$, finalmente, que la marca asociada a alta SF obtuvo una evaluación más positiva. Se valora el impacto del estudio en la operacionalización del atractivo físico, la identificación de estímulos incondicionados y las ventajas del procedimiento en la evaluación de comunicaciones persuasivas.

Palabras clave: Psicología Evolucionista, Condicionamiento Clásico, actitudes del consumidor, simetría facial.

Artículo de investigación. http://dx.doi.org/10.15332/s1794-9998.2016.0001.03

Correspondencia: Danilo Zambrano, Universidad de Ibagué, Colombia. Dirección postal: calle 77 No. 85-36, barrio La Granja. Correo electrónico: danilozambrano@psicologos.com 


\section{Abstract}

In a sample of 34 university students (17 of each sex and on average 21 years old) at the Universidad de lbagué, we evaluated the effect of facial symmetry (FS) in a Classical Conditioning of Attitudes experiment, using a simultaneous procedure where three logos were used as conditioned stimuli (Xua, Xue, Xuo) and faces of varying symmetry were used as unconditioned stimuli. We observed a higher rating of attractiveness of faces with high FS. We also observed a change in ratings of each logo before and after the conditioning procedure, and finally, the logo associated with a high FS obtained significantly higher scores after conditioning (than the logos associated with less FS). The impact of our study is that we operationalized physical attractiveness, identifying an unconditioned stimulus (facial symmetry as an index of physical attractiveness) and demonstrated the advantage of our conditioning procedure in establishing and changing the attitudes of consumers in persuasive communications.

Keywords: Evolutionary Psychology, Classical Conditioning, attitudes of consumer, facial symmetry.

\section{Introducción}

El uso de personas atractivas en la publicidad está documentado y es objeto de polémicas acerca de sus efectos no deseados (Bower, 2001; Saad, 2004); sin embargo, el empleo frecuente de fuentes atractivas por quienes diseñan y ejecutan este tipo de comunicaciones persuasivas (CP), se basa en el apoyo empírico que recibe el atractivo como recurso persuasivo (Baker y Churchill, 1977; Chaiken, 1979; Joseph, 1982).

Son diversas las aproximaciones que explican el por qué el atractivo de la fuente es un recurso que contribuye efectivamente en las CP (Trampe, Stapel, Siero y Mulder, 2010); una de ellas es la Psicología Social (PS). Las primeras incursiones de la psicología a las $C P$ se realizaron a finales del siglo XIX y comienzos del siglo XX (Benjamin, 2004). No obstante, en la PS (Briñol y Petty, 2012), los aportes más conocidos son aquellos difundidos cerca a la primera mitad del siglo XX en el tema de la adquisición y cambio de actitudes (Hovland, Janis y Kelley, 1953) y, precisamente, por esta época es cuando el atractivo de la fuente se constituye en un objeto de análisis sistemático.
Unas décadas después, se da un paso definitivo, al definir el atractivo como una evaluación (Walster y Walster, 1963), y por consiguiente, con esa noción es incorporado a uno de los desarrollos teóricos y metodológicos más avanzados de esta especialidad: las actitudes -ver, por ejemplo, Dion, Berscheid y Walster, (1972) y Eagly, Ashmore, Makhijani y Longo (1991), o Langlois, Kalakanis, Rubenstein, Larson, HaUam y Smoot (2000)-.

Solo recientemente y apoyados por los desarrollos de la Psicología Evolucionista y las Neurociencias (Cacioppo, Berntson y Decety, 2010), los psicólogos sociales comenzaron a identificar rasgos que hacen a una persona físicamente atractiva y a plantear formulaciones con valor explicativo y predictivo (Etcoff, 1999; Grammer, Fink, Moller y Thornhill, 2003). Una de las categorías del atractivo beneficiadas con estas formulaciones fue el atractivo facial (Aharon, Etcoff, Ariely, Chabris, O'Connor y Breiter, 2001; Fink y Penton-Voak, 2002; Zebrowitz y Montepare, 2008).

Sin embargo, el impacto de dichas formulaciones en distintos temas de la PS, no se refleja con la misma intensidad en las aproximaciones psicoso- 
ciales que se ocupan del estudio, diseño, ejecución y evaluación de las CP (Colarelli y Dettman, 2003; Vyncke, 2011).

Este estudio se propuso considerar una de esas formulaciones acerca del atractivo facial, conocida como la teoría de la simetría facial y someterla a prueba en un diseño donde el rostro de una modelo se constituye en el recurso persuasivo central. Para ello, utilizó un paradigma experimental que es eficaz en la investigación de diversos fenómenos del comportamiento, en numerosas especies y bajo distintos niveles de análisis (Kandel, Abrams, Bernier, Carew, Hawkins y Schwartz, 1983), denominado el Condicionamiento Clásico o Condicionamiento Pavloviano (Schachtman y Reilly, 2011).

Uno de los paradigmas más utilizados en la psicología (Rescorla, 1988), tanto en la investigación básica como en la aplicada, y una tradición en el estudio de la adquisición y cambio de actitudes mediante CP (Schachtman, Walker, Fowler, 2011; Pornpitakpan, 2012)

\section{El atractivo de la fuente y la simetría facial}

Como se señaló, el atractivo de la fuente es una estrategia común en el diseño de CP y sus efectos son ampliamente aceptados y apoyados empíricamente (Baker y Churchill, 1977; Chaiken, 1979; Trampe et al., 2010; Vyncke, 2011). La relevancia del atractivo como recurso para influir en las actitudes de la audiencia ha conducido a esfuerzos para identificar qué es lo que hace a una persona atractiva $y$, en consecuencia, a sugerir varios tipos y taxonomías acerca de cuándo una persona es atractiva -ver, por ejemplo, Briñol, de la Corte y Becerra (2001)-. Sin embargo, todavía está pendiente una investigación sistemática acerca de cuándo y cómo una persona resulta atractiva físicamente a la audiencia (Brownlow, 1992).

En el contexto de la PS existen diversas aproximaciones sobre la belleza, desde aquellas relativistas hasta aquellas que postulan unos universales acerca del atractivo físico (Zebrowitz, 1997). Específicamente, las formulaciones de la Psicología Evolucionista y las Neurociencias en la PS han in- fluido en la expansión de explicaciones universalistas del atractivo físico, tales como las razones cintura-cadera (mujeres), y cintura-hombros (hombres), la cara de bebé, etc. (Gould, 1979; Etcoff, 1999).

En el caso particular de los rostros atractivos, los resultados consistentes acerca de la valoración positiva que recibe la cara promedio pueden ser explicados por la noción de Simetría Facial (SF) (Zebrowitz, 1997). De acuerdo con las ideas planteadas desde la PE, la SF actúa como señal de salud, calidad genética y potencial reproductivo (Buss, 2007; Buss, 2013) y está relacionada positivamente con muchos factores que reflejan salud, calidad reproductiva y mejor disposición atlética y de talentos (Rentschler, Jüttner, Unzicker, y Landis, 1999; Baudouin y Tiberghien, 2004; Gangestad y Scheyd, 2005; Wade, 2010). Una cualidad que es observada en miembros de varias especies incluyendo a los humanos (Shepherd y Bar, 2011).

Con el fin de ampliar las aplicaciones de las formulaciones evolucionistas al diseño de CP eficaces, esta investigación utilizó la hipótesis de la SF para proponer una aproximación objetiva y sistemática al tema del atractivo físico de la fuente. Es importante resaltar que la elección por una aproximación basada en la SF se apoya en los resultados observados por Peters, Rhodes y Simmons (2007) respecto a la primacía de las evaluaciones del rostro sobre las de otros atributos físicos de las personas.

La SF es entendida como el grado de equivalencia cuantitativa entre las dos partes laterales del rostro (derecho e izquierdo), es decir, la uniformidad en la distribución y dimensiones de los rasgos del lado izquierdo con los del lado derecho (Jones y DeBruine, 2007). Específicamente, este estudio midió la SF bilateral del eje horizontal, dado que ha sido la más utilizada en los estudios empíricos sobre el tema (Hume y Montgomery, 2001; Jones et al., 2001).

Hume y Montgomery (2001) exponen que la SF es un buen indicador de estabilidad en el desarrollo en la calidad genética y, en consecuencia, un predictor del nivel del atractivo físico. En sus estudios 
encontraron una relación negativa entre las calificaciones del atractivo facial (rostros de mujeres) y el índice de masa corporal y los problemas de salud en las mujeres; también comprobaron, al igual que Jones, Little, Penton-Voak, Tiddeman, Burt y Perrett (2001) y Rhodes (2006), que las calificaciones de atractivo alto eran atribuidas a los rostros simétricos.

En el contexto de la SF, la salud y el potencial reproductivo, algunas hormonas femeninas como el estrógeno y el estradiol juegan un papel importante; por ejemplo, Jasienska, Ziomkiewicz, Ellison, Lipson y Thune (2006) hallaron una relación directa entre simetría y niveles de estrógeno, y Cetinkaya, Dural y Gulbetekin (2010) encontraron que los rostros de las mujeres se tornan más simétricos en la fase de ovulación y son calificadas como más atractivas.

Adicionalmente, la SF también provee valiosa información sobre rasgos psicológicos o comportamentales (Fink, Neave, Manning y Grammer, 2005), en concordancia con el fenómeno psicosocial: el "estereotipo... lo bello es bueno" (Dion, Berscheid, y Walster, 1972; Nisbett y Wilson, 1977; Creusen, Veryzer y Schoormans, 2010).

Sin embargo, las reacciones provocadas por las caras atractivas no están bien establecidas; por ejemplo, Mientras que Noor y Evans (2003) mostraron que las caras menos simétricas fueron evaluadas con rasgos de personalidad menos agradables, Principe y Langlois (2011) hallaron una relación inversa entre valores del atractivo y la respuesta facial muscular de desagrado; Van Valen y Macre (2004), señalaron que no siempre las caras poco atractivas provocan una evaluación negativa. Incluso, Portales, Gil y Beltrami (2009) encontraron que las mujeres parecen más hábiles en detectar las diferencias en la SF que los hombres.

Una forma usual de registrar la SF es la Asimetría Fluctuante (AF), la AF es definida como la desviación estándar relativa a la simetría de un atributo o carácter bilateral (Cuervo, 2000; Graham, Raz, Hel-Or y Nevo, 2010) y parte del supuesto que la diferencia de los lados derecho e izquierdo del rostro (en sus mediciones) deben acercarse a cero (baja AF) para ser considerados con alta simetría.
Wade (2010) expone que la medición de la AF revela la simetría de un rostro humano y que tal armonía está asociada con el nivel de atractivo. Adicionalmente, según la teoría parasitaria de la selección sexual, los humanos desarrollaron durante su historia natural una serie de módulos para detectar y preferir una baja AF y por consiguiente evitar una alta AF en la potencial pareja (Grammer y Thornhill, 1994). Jasienska et al. (2006) en un estudio con 171 mujeres polacas, encontraron que el grado de AF está relacionado con los niveles de la hormona estradiol que se encuentra en los ovarios y, como lo señalan, Lipson y Ellison (1996) y Durante y Li (2009), esta hormona se asocia a la probabilidad de concepción o potencial reproductivo.

En consecuencia, una mayor o menor $\mathrm{AF}$ en el rostro es, desde una lógica evolucionista, un indicador importante sobre las calidades de la potencial pareja sexual y, visto desde la perspectiva del Condicionamiento Clásico, la mayor o menor AF son estímulos que poseen una significación biológica y que por lo tanto pueden actuar como El al provocar una Respuesta Incondicionada (RI) de carácter evaluativo, con una dirección (positiva o negativa) y una intensidad (mayor o menor) perfectamente identificables y susceptibles de cuantificación con instrumentos como, por ejemplo, una escala de actitudes (Osgood, Suci y Tannenbaum, 1957; Hughes, 1967).

Desde la emergencia del paradigma del Condicionamiento Clásico (CC) (Windholz, 1989), el que un estímulo posea una significación biológica es un criterio fundamental para definirlo como Estímulo Incondicionado (EI) y, por consiguiente, para ser utilizado como reforzador en un procedimiento donde este es apareado con otro estímulo, el Estímulo Neutro (EN). Sin embargo, la identificación de cuándo un estímulo es un El ha estado sujeto a caracterizaciones que carecen de valor heurístico y predictivo dada la dificultad en alcanzar una definición objetiva de sus propiedades distintivas (Schoenfeld, 1995; Berridge, 2001).

Esta limitación predictiva del CC se ha reflejado en las distintas extensiones y aplicaciones que se hacen de este paradigma (Schachtman y Reilly, 
2011); y, por supuesto, en la psicología de las CP en donde se conoce como Condicionamiento Clásico de Actitudes (CCA) (Schachtman, Walker y Fowler, 2011; Pornpitakpan, 2012).

\section{El condicionamiento clásico de actitudes en las comunicaciones persuasivas}

Las actitudes es uno de los conceptos más tradicionales de la PS (Crano y Prislin, 2006) y uno de los temas más utilizados en las disciplinas y especialidades que tienen como tarea describir, explicar, predecir y controlar el comportamiento del consumidor y el de las CP acerca de marcas y productos que constituyen un mercado específico.

Aunque se han formulado diferentes nociones acerca de la actitud y su naturaleza -innata o adquirida (Tesser, 1993; Olson, Vernon, Harris y Jang, 2001)-, aquí se acoge la definición hoy dominante en la PS, donde se formula que es una respuesta evaluativa con una dirección - positiva o negativa- y una intensidad - desde baja hasta alta- y, que de acuerdo con Ajzen (2001), resume las calificaciones que un individuo hace a partir de dimensiones bipolares como "bueno-malo, peligroso-benéfico, placentero-displacentero, agradable-desagradable" (p. 28).

El frecuente uso de la noción de actitud, en campos de conocimiento como la PS y la Psicología de la Publicidad, se origina en su capacidad para pronosticar un gran número de cursos de acción por los que los individuos optan frente a un conjunto, amplio, de alternativas de consumo (Horcajo, Briñol y Petty, 2010).

Los expertos reconocen que si bien la relación actitud-conducta es bastante compleja (Ajzen y Fishbein, 2005), las actitudes siguen siendo una herramienta fundamental para el logro de las metas que se fijan; una muestra de ello, son las cifras que anualmente se invierten en la investigación y en los esfuerzos por inducir actitudes favorables hacia sus marcas y productos.
Los psicólogos sociales en su esfuerzo por identificar las variables responsables del origen y cambio de las actitudes han formulado y evaluado varias propuestas, una de esas propuestas que se origina en las aproximaciones al aprendizaje de origen conductista, es el CCA (Olson y Fazio, 2001). La trayectoria del CCA se puede seguir desde mediados del siglo XX (Staats y Staats, 1958) y, desde entonces, ha mostrado ser un procedimiento eficaz para la adquisición y modificación de actitudes, independientemente si estos cambios se originan en procesos intencionales o no, conscientes o no, etc. Las revisiones muestran al CCA como un área de investigación diversificada en términos de los estímulos y condiciones experimentales (Froufe y Sierra, 1998). En el caso específico de los estímulos incondicionados utilizados, se ha recurrido a eventos propios de los canales visual y auditivo $y$, entre ellos, el investigado en este estudio: el atractivo físico de la fuente.

La utilización de fuentes atractivas a partir de los criterios establecidos por las formulaciones evolucionistas y neurocientíficas, tiene un propósito adicional que está relacionado con la identificación de universales de la belleza y, en consecuencia, el de contar con elementos de juicio empíricamente validados para sugerir pautas a quienes diseñan y evalúan CP globales (Ellis y $\mathrm{He}, 2011)$.

El CCA a partir del atractivo de la fuente, requiere de un $\mathrm{EI}$ - un evento que provoca regularmente la respuesta estudiada - y que, en este caso particular, corresponde a un rostro bello; una RI -la respuesta estudiada que es provocada regularmente por el El- y que consiste en la evaluación positiva o actitud del sujeto; un EN -un evento que no provoca regularmente la actitud estudiada- y que, en este tipo de investigaciones, suele hacer referencia a una marca o producto; un procedimiento de apareamiento de los dos estímulos -El y EN-; y, finalmente, cuando el EN comienza a provocar la actitud estudiada, se habla de un efecto de condicionamiento, y el estímulo, anteriormente neutro, comienza a denominarse Estímulo Condicionado (EC) y a la actitud observada ante el EC, se le conoce como Respuesta Condicionada (RC). 
Un estudio de CCA donde se emplea el atractivo físico de la fuente es el de Cruz (2008). Su investigación se basó en formulaciones evolucionistas acerca de la razón cintura-cadera como una variable del atractivo de la fuente que provee de información con significación biológica (Henss, 2000; Jasienska, Ziomkiewicz, Ellison, Lipson y Thune, 2004). En dicho estudio, se utilizó como El tres valores de la razón cintura-cadera (0.7, 0.8 y 0.9), como primer resultado se observó que los participantes mostraron una mayor preferencia por la relación cintura-cadera de menor valor, acorde a las formulaciones evolucionistas. Adicionalmente, el investigador encontró evidencia sobre la capacidad que como El tuvo la relación cintura-cadera para transferir la evaluación provocada a la marca con que fue apareada.

Siguiendo la línea de pensamiento de utilizar como El un estímulo con probada significación biológica, como el atractivo físico de la fuente y, por otro lado, haciendo uso de las formulaciones evolucionistas acerca de la SF como un indicador, perfectamente cuantificable, de atractivo, el objetivo de este estudio fue aportar evidencia empírica sobre la formación y cambio de actitudes en los consumidores a través de un procedimiento de CC, utilizando tres valores (alta, media y baja) de SF como El y, con ello, brindar no solo apoyo empírico a otro de los indicadores de atractivo planteados por la Psicología Evolucionista, sino también contribuir con observaciones acerca de cuándo y cómo una fuente es atractiva físicamente -en el tema de las $\mathrm{CP}-\mathrm{y}$ cómo se puede identificar un El -en el terreno del CCA-.

En la siguiente sección se exponen las predicciones que se plantearon.

\section{Hipótesis}

1. Los rostros con alta SF serán mejor evaluados por los participantes que los rostros con media y baja simetría.

2. Las evaluaciones realizadas a las marcas, posterior al procedimiento de apareamiento con los distintos valores de SF, serán diferentes a las registradas en la medición previa al condicionamiento.
3. La marca asociada a los rostros con alta SF provocará en los participantes una evaluación positiva y mayor que la realizada a las marcas asociadas a los rostros con media y baja simetría.

\section{Método}

\section{Tipo de estudio}

Estudio de tipo experimental de un grupo al que se le aplicó, de manera aleatoria, cada uno de los tres valores de la variable independiente $-\mathrm{SF}$ alta, media y baja - y con medidas pretest/postest para evaluar los respectivos efectos de la variable independiente sobre la variable dependiente -la actitud o evaluación a la marca-.

\section{Sujetos}

Se seleccionaron mediante un muestreo por conveniencia en función de la edad y la orientación sexual, 34 estudiantes de la Universidad de Ibagué (17 hombres y 17 mujeres) heterosexuales, con edades comprendidas entre los 18 y 23 años $(M=21,0)$.

\section{Instrumentos y materiales}

- Cuatro rostros femeninos, cada uno con 3 valores de SF (alta, media y baja), para un total de 12 estímulos incondicionados.

- Tres marcas diseñadas por Cruz (2008) -Xua, Xue y Xuo-.

- Tres escalas de diferencial semántico cuyos polos fueron agradable $(+6)$ /desagradable $(-6)$; bonita $(+6) /$ fea $(-6)$ e indeseable $(-6) /$ deseable $(+6)$.

- El programa ImageJ versión1.46 para Windows de 32 bit (desarrollado por US National Institutes of Health, disponible en http://rsb.info.nih. gov/ij).

- Una cámara digital a color Canon EOS REBEL T3i.

- El programa Adobe Photoshop CS6. 
- Computador HP pro 3400 series mt, con procesador intel (R) Corel (TM) 3-2 100 CPU @3. 106hz, y pantalla LCD Samsung a color de 19 pulgadas.

- Computador Compaq Presario CQ40 Notebook PC.

\section{Procedimiento}

El procedimiento implicó dos fases: a) diseño de los estímulos incondicionados y b) aplicación del experimento.

\section{a. Diseño de los estímulos incondicionados}

\section{Etapa 1:obtención de las fotografías (estímulos incondicionados)}

Se tomaron 5 fotografías a 4 mujeres (18-23 años de edad, $M=21$ años) con una cámara digital con flash para evitar inconvenientes a partir de sombras en los rostros. Cada fotografía se tomó desde un trípode fijo con variación vertical a una distancia de 2 metros de la modelo y sin utilizar el zoom, solo hubo variación en el enfoque de manera manual. Antes de comenzar la sesión fotográfica, a cada participante se le solicitó que se removiera el maquillaje y los accesorios -p.e. piercing-. Igualmente se les solicitó que se peinaran hacia atrás dejando ver las orejas. Para tomar el registro fotográfico, a las modelos se les insistió que tuviesen una expresión facial neutra, con los labios juntos pero sin apretarlos y que mantuviesen una postura erguida. Una vez obtenidas las imágenes se procedió a seleccionar las mejores imágenes a utilizar.

\section{Etapa 2: modificación de las fotografías}

Se trataron las fotografías con el programa Adobe Photoshop CS6 para cambiarlas a blanco y negro, se estandarizó la superficie y el tono de la piel de los rostros, luego se hicieron 2 versiones espejo del lado derecho e izquierdo de cada uno y se seleccionaron aquellas que más coincidían, viéndose así más naturales; posteriormente, se modificaron los ojos, la nariz, los labios y el contorno del rostro para lograr versiones de baja, media y alta simetría. Igualmente, se difuminó el cabello y las orejas (ver figura 1). De esta forma se llegó a obtener tres imágenes por cada una de las cuatro modelos, para un total de doce retratos, con una resolución de $1784 \times 2254$ pixeles, una profundidad de 24 bits y una resolución de impresión de 106 ppp.

\section{Etapa 3: medición de la asimetría}

Todas las mediciones de la SF se realizaron con el programa ImageJ. La medición de la simetría se hizo a través de 12 puntos apareados bilateralmente (ver figura 2) del eje horizontal de los rostros, los cuales han demostrado una alta repetitividad (Hume y Montgomery, 2001; Jones et al., 2001).

Figura 1.

Versiones de baja, media y alta simetría en el rostro de una de las modelos.

SIMETRIA

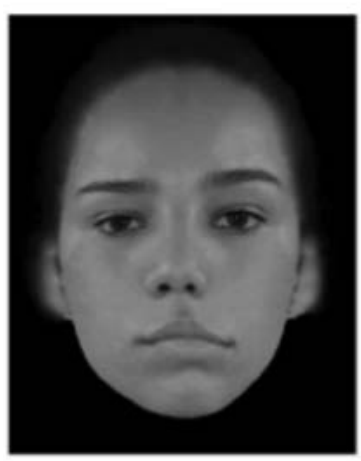

Baja

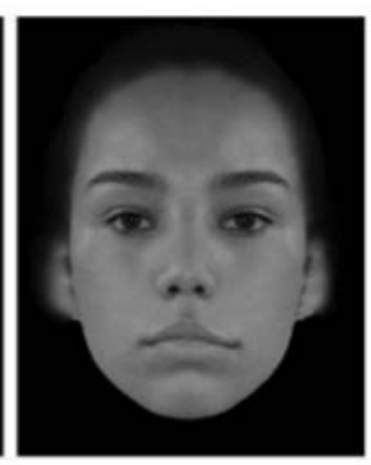

Media

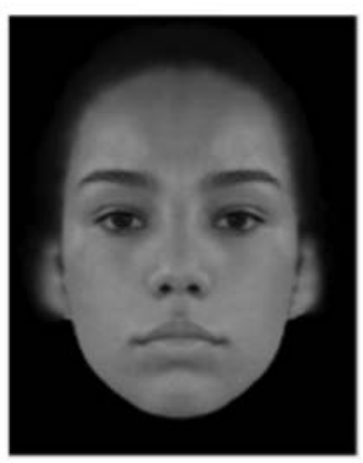

Alta 


\section{Etapa 4: validación de los estímulos}

El objetivo de esta fase fue determinar si los estímulos creados (rostros femeninos) en el programa Adobe Photoshop CS6, reflejaban una baja, media y alta simetría. Se realizó con 20 sujetos heterosexuales (10 hombres y 10 mujeres, con edades entre 18 y 24 años, $M=21$ años), estudiantes de la Universidad de Ibagué de diferentes programas académicos de pregrado.

Las imágenes fueron exhibidas de manera individual, en un computador Compaq Presario mediante una serie de diapositivas con un tamaño de 19 $\mathrm{cm} \times 15,04 \mathrm{~cm}$. A los participantes se les solicitó que ante cada imagen respondieran 2 escalas tipo Likert de 1-10; la primera escala midió el atractivo ( $1=$ No es atractiva $/ 10=$ Es muy atractiva) y la segunda escala estimó la simetría ( $1=$ No es simétri$\mathrm{ca} / 10=$ Es muy simétrica) - un procedimiento ya ha sido aplicado para la evaluación de belleza (ver Rhodes, Proffitt, Grady y Sumich, 1998)-.

Figura 2.

Puntos utilizados para medir la asimetría en los rostros. El plano de la simetría (PS) se midió promediando la media de la distancia entre los puntos (por ejemplo: la media de P1 y P2). Cada característica del rostro de derecha e izquierda, fue medida de la siguiente manera: ojo exterior (PS-P1, P2-PS), ojo interno (PS - P3, P4 - PS), ancho del ojo (P3 - P1, P2 - P4), cara (PS - P5, P6 - PS), nariz (PS - P7, P8 - PS), mandíbula (PS - P9, P10 - PS) y boca (PS - P11, P12 - PS).

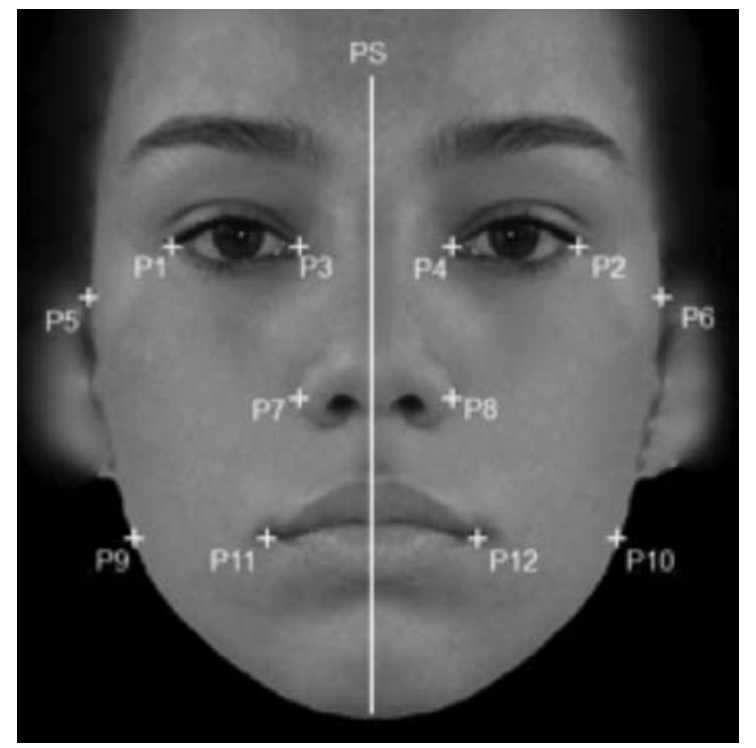

Se aplicó un análisis de medias a las evaluaciones obtenidas sobre las fotos de las modelos y los resultados mostraron diferencias en las calificaciones, y que tales diferencias se orientaron en función tanto del grado de simetría como del nivel de atractivo, simetría baja 2.64 (0.44), simetría media $X=4.89(0.69)$ y simetría alta $X=7.78(0.41)$; atractivo bajo $X=3.13(0.48)$, atractivo medio $X=4.75(0.32)$ y atractivo alto $X=6.06(0.81)$.

A partir de estos resultados, se tomó la decisión de utilizar como estímulos incondicionados las fotos de las cuatro modelos.

\section{b. Aplicación del experimento}

Se realizó una aplicación individual en un cubículo del laboratorio de psicología de la Universidad de Ibagué. Se inició con un saludo y agradecimiento por participar en el estudio; enseguida, uno de los investigadores ofreció una breve descripción de las condiciones experimentales y las conductas esperadas durante el procedimiento; posteriormente, se entregó el consentimiento, se le concedió un tiempo para leerlo y resolver dudas. Una vez aceptado y firmado el consentimiento, se procedió a dar las instrucciones requeridas para comenzar con el experimento, el cual se estructuró en tres etapas, siguiendo el procedimiento de Cruz (2008).

\section{Etapa 1: precondicionamiento o anclaje y pretest}

A cada participante se le presentó la secuencia de los 12 estímulos incondicionados con una duración de dos segundos por estímulo. Este procedimiento, conocido como anclaje, obedece a un control experimental que procura evitar efectos de techo y suele realizarse antes de cualquier tarea de evaluación. Después, para obtener las medidas pretest se exhibieron nuevamente los 12 estímulos incondicionados durante 15 segundos cada uno, y se les solicitó a los participantes calificar cada estímulo a través de tres escalas de diferencial semántico.

Posteriormente, el proceso de anclaje y pretest descrito se repitió con todos los participantes, 
pero en este caso para la respectiva evaluación de cada uno de los logos - Xue, Xuo y Xua-.

\section{Etapa 2: condicionamiento}

Luego del anclaje, cada participante fue expuesto a 12 pares de estímulos (4 rostros para cada una de las 3 marcas) organizados de la siguiente manera: 4 rostros con baja simetría apareados con la marca Xuo, 4 rostros con simetría media apareados a la marca Xua y 4 rostros con alta simetría apareados con la marca Xue. La presentación de los pares estimulares se realizó bajo un procedimiento simultáneo, y la extensión del intervalo inter-ensayos se distribuyó aleatoriamente en un lapso de tiempo que varió desde los 5 segundos hasta los 15 segundos, en pausas correspondientes a cifras en múltiplos de cinco.

\section{Etapa 3: postcondicionamiento}

Culminada la etapa de condicionamiento, se solicitó a los participantes que evaluaran nuevamente las marcas con las 3 escalas utilizadas en la etapa de precondicionamiento.

Una vez finalizado el experimento, con los datos consolidados en una hoja electrónica, se procedió a realizar la conversión de las respuestas de los participantes, que estaban en valores de $-6 \mathrm{a}+6 \mathrm{a}$ valores entre 1 y 13 con la siguiente fórmula: $X+6$ $y$, una vez realizado el ajuste, se realizó el tratamiento estadístico de los datos. Los resultados y su análisis se presentan en la siguiente sección.

\section{Resultados}

Como se señaló en su momento, las predicciones de esta investigación fueron:

1. Los rostros con alta SF serán mejor evaluados por los participantes que los rostros con media y baja simetría.

2. Las evaluaciones realizadas a las marcas, posterior al procedimiento de apareamiento con los distintos valores de SF, serán diferentes a las registradas en la medición previa al condicionamiento.
3. La marca asociada a los rostros con alta SF provocará en los participantes una evaluación positiva y mayor que la realizada a las marcas asociadas a los rostros con media y baja simetría.

En cuanto a la primera hipótesis, los resultados en la tabla 1 muestran que los participantes tuvieron una mayor preferencia por los rostros con alta simetría, los cuales arrojaron mayores puntajes en las cuatro modelos utilizadas. Adicionalmente, los datos revelan que no existen grandes diferencias en las evaluaciones entre-modelos en función de cada una de las simetrías.

Respecto al análisis de las medias en las evaluaciones realizadas a las marcas durante las etapas previa y posterior al condicionamiento -hipótesis $2-$, como se puede observar en la tabla 2 , en la etapa de precondicionamiento, no hubo un sesgo de favorabilidad o preferencia por alguna marca en especial y, por consiguiente, Xua, Xue y Xuo se comportaron como estímulos neutros.

Adicionalmente, como se observa en la tabla 2, las medias de las valoraciones de favorabilidad de las marcas antes y después del condicionamiento muestran diferencias y, por consiguiente, es posible prever un efecto del apareamiento sobre la evaluación de los sujetos hacia las marcas.

En la misma línea, la tabla 2 señala que Xua y Xue las marcas apareadas con las SF media y alta respectivamente, mostraron un cambio positivo entre las dos medidas; mientras que la marca Xuo, apareada con la SF baja, tendió a presentar un cambio en la dirección opuesta. Esto apoya la tercera hipótesis que pronosticaba un efecto de mayor favorabilidad en aquella marca que fue apareada con la imagen con SF alta.

Finalmente, se analizaron los datos recolectados de las calificaciones de agradable, bonita y deseable de las etapas pre y post en el programa SPSS versión 15.0, y se realizó la prueba T para muestras relacionadas.

Como se observa en la tabla 3 , las marcas tuvieron un cambio en las calificaciones de las etapas pre y post. La marca Xua que fue asociada a la simetría media tuvo diferencias estadísticamente 
significativas en las puntuaciones de dos de las tres escalas empleadas, bonita y deseable; por su parte, la marca Xue apareada al rostro con alta simetría, mostró un cambio estadísticamente significativo $p<.05$ en las tres escalas (agradable, bonita y deseable).

Tabla 1.

Medias para las tres simetrías de las cuatro modelos.

\begin{tabular}{llccc}
\hline Modelo & Descriptivos & Simetría baja & Simetría media & Simetría alta \\
\hline Modelo 1 & $\bar{X}$ & 6,61 & 7,72 & 8,26 \\
\hline & Desv. Típica & 0,47 & 0,46 & 0,56 \\
\hline Modelo 2 & $\bar{X}$ & 6,01 & 7,07 & 8,08 \\
\hline & Desv. Típica & 0,35 & 0,46 & 0,62 \\
\hline Modelo 3 & $\bar{X}$ & 6,04 & 7,86 & 8,69 \\
\hline & Desv. Típica & 0,39 & 0,53 & 0,63 \\
\hline Modelo 4 & $\bar{X}$ & 6,66 & 7,56 & 8,20 \\
\hline & Desv. Típica & 0,63 & 0,66 & 0,36 \\
\hline
\end{tabular}

Tabla 2.

Medias de las marcas durante las etapas pre y post.

\begin{tabular}{llccc}
\hline Medida & Descriptivos & Marca 1: Xuo & Marca 2: Xua & Marca 3: Xue \\
\hline 1 “Pre” & $\bar{X}$ & 7,89 & 7,15 & 7,34 \\
\hline & Desv. Típica & 0,41 & 0,77 & 0,79 \\
\hline 2 “Post” & $\bar{X}$ & 7,73 & 8,01 & 8,78 \\
\hline & Desv. Típica & 0,78 & 0,35 & 0,76 \\
\hline
\end{tabular}

$\mathrm{N}=34$

Tabla 3.

Resultados de la Prueba t para las calificaciones de las marcas en las etapas pre y post.

\begin{tabular}{lllllllll}
\hline 95 \% IC & & & & & & & & \\
\hline Logo & Calificativo & M & DE & ET & LI & LS & t(33) & $\begin{array}{c}\text { Sig } \\
\text { (bilateral) }\end{array}$ \\
\hline Xuo & Agradable &,- 21 & 1,86 &, 32 &,- 85 &, 44 &,- 65 &, 52 \\
\hline & Bonita &, 12 & 1,95 &, 33 &,- 56 &, 80 &, 35 &, 73 \\
\hline & Deseable &, 59 & 1,73 &, 30 &,- 01 & 1,19 & 1,99 &, 05 \\
\hline Xua & Agradable &,- 44 & 2,40 &, 41 & $-1,28$ &, 40 & $-1,07$ &, 29 \\
\hline & Bonita &,- 82 & 2,02 &, 35 & $-1,53$ &,- 12 & $-2,38$ &, 02 \\
\hline & Deseable & $-1,32$ & 2,51 &, 43 & $-2,20$ &,- 45 & $-3,08$ &, 00 \\
\hline Xue & Agradable & $-1,41$ & 2,24 &, 39 & $-2,20$ &,- 63 & $-3,67$ &, 00 \\
\hline & Bonita & $-1,32$ & 1,82 &, 31 & $-1,96$ &,- 69 & $-4,24$ &, 00 \\
\hline & Deseable & $-1,56$ & 1,99 &, 34 & $-2,25$ &,- 87 & $-4,57$ &, 00 \\
\hline
\end{tabular}

Nota. IC= intervalo de confianza; LI: límite inferior; LS: límite superior. 
En síntesis, los resultados apoyan la hipótesis 1 en el sentido de que los rostros con alta SF fueron mejor evaluados por los participantes que los rostros con media y baja simetría; igualmente, el análisis permite confirmar la hipótesis 2, la cual pronosticaba un cambio en las evaluaciones realizadas a los logos antes del procedimiento y aquellas producidas después del mismo y, por último, apoyan empíricamente la tercera hipótesis que predecía una mayor y más positiva evaluación hacia la marca asociada a los rostros con alta simetría que la mostrada hacia los logos asociados a los rostros con simetría media y baja.

\section{Discusión}

Continuando con la labor de identificar atributos dimensionales que hacen al atractivo físico un estímulo biológicamente significativo en el CCA (Cruz, 2008), este estudio experimental valoró la SF como una variable que permite predecir con éxito cuándo un evento operará como El.

El escoger la simetría como una variable que permita identificar EEll e, incluso, ofrecer una explicación acerca del porqué de su significación biológica, es el resultado de una serie de estudios que señalan la predilección de una gran variedad de especies por objetos y otros seres vivos que poseen tal cualidad (Shepherd y Bar, 2011).

Una razón importante para la escogencia de la SF en este esfuerzo por vincular los desarrollos de la Psicología Evolucionista, al CCA y, por consiguiente, al diseño de comunicaciones persuasivas, proviene de los hallazgos de Peters, Rhodes y Simmons (2007), que advierten sobre el predominio de la evaluación del atractivo facial sobre otros atributos del atractivo físico de las personas.

En esta línea, esta investigación contrastó empíricamente las predicciones evolucionistas, donde se enuncia que diversos grados de simetría conducen a diferentes valores en las evaluaciones de atractivo de las caras; los resultados obtenidos otorgan un apoyo empírico a tal hipótesis $y$, por consiguiente, a las formulaciones que en este sentido ya se han realizado por otros investigadores del tema (Gangestad y Scheyd, 2005; Rhodes, 2006 y Wade, 2010).

Las diferencias estadísticamente significativas entre las evaluaciones realizadas por los participantes a los tres niveles de SF e independientemente de la modelo calificada, advierten sobre la capacidad de estos atributos dimensionales de provocar una respuesta evaluativa diferente para cada uno de los grados de simetría tasados durante el procedimiento. Tales resultados constituyen un apoyo empírico para aquellas formulaciones evolucionistas que explican las preferencias por rostros simétricos dada su capacidad informativa acerca del valor reproductivo, condición de salud, etc. (Buss, 2007).

Por otro lado, al encontrar estos resultados en población colombiana, estos hallazgos otorgan un soporte empírico indirecto a las formulaciones que insisten en las ventajas de utilizar en las comunicaciones persuasivas modelos que poseen "rasgos" o "atributos dimensionales" universales de atractivo (Ellis y He, 2011).

Adicionalmente, este estudio en comparación con la investigación realizada por Cruz (2008), tuvo resultados más consistentes en las evaluaciones de las distintas modelos que poseen los mismos niveles de simetría, ya que las puntuaciones de las evaluaciones entre los rostros de las modelos fueron similares, es decir no tuvieron mayor variación al interior de cada uno de los niveles (simetría baja, media y alta) debido a que se realizó un mayor control de variables (por ejemplo, manteniendo constante el fondo en todas las imágenes) y, por último, al hecho de que se trataban de modelos que comparten más rasgos fisonómicos que aquellas utilizadas en el estudio citado.

La importancia de la simetría en el ámbito del consumidor no se limita a las decisiones involucradas en el diseño de comunicaciones persuasivas donde se recurre a personas, varios estudios empíricos muestran que esta preferencia también se expresa cuando se trata de elegir entre objetos $y$, lo más interesante, conduciendo a fenómenos psicosociales semejantes a los observados con personas atractivas, como en los casos del estereotipo "lo bello es bueno" (Dion, Berscheid y Walster, 
1972) y el efecto halo (Fink, Neave, Manning y Grammer, 2005; Creusen, Veryzer y Schoormans, 2010).

Del mismo modo, en este estudio, se procuró brindar apoyo empírico a los procedimientos de CCA y, efectivamente, esa meta se logra como lo señalan las diferencias encontradas en las evaluaciones de las marcas entre las medidas antes y después del apareamiento con los estímulos.

Igualmente, estos resultados son importantes para esta línea de investigación que persigue vincular los desarrollos evolucionistas acerca del atractivo físico con preguntas de investigación básica y aplicada, tanto en la psicología de las comunicaciones persuasivas como en el CCA, y que empezó con el estudio de la razón cintura-cadera como variable que predice cuándo un evento actuará como El (ver Cruz, 2008).

Aunque se ha señalado con suficiencia las ventajas del paradigma experimental del CCA en las cuestiones básicas, este estudio se constituye en un soporte más, no solo para el CC como un paradigma exitoso en la psicología básica sino también en los asuntos aplicados (Rescorla, 1988), al ofrecer un procedimiento para la evaluación de las comunicaciones persuasivas en sus diferentes etapas -en el diseño y prueba de bocetos como durante el lanzamiento de la campaña y posteriormente a su ejecución-.

Adicionalmente, con este experimento se buscó probar las predicciones acerca de la SF como un estímulo biológicamente relevante $\mathrm{y}$, en consecuencia, su utilidad como El en un procedimiento de Condicionamiento Clásico de Actitudes. Los resultados obtenidos otorgan un apoyo empírico a las formulaciones evolucionistas que presentan a la simetría en el rostro como una dimensión biológicamente significativa (Buss, 2007; Wade, 2010; Jasienska et al., 2006), lo que a su vez hace que el atributo de la SF pueda ser considerado en el momento de elegir el El al que se le va a aparear una marca o producto.

Los resultados observados muestran que la SF alta resultó ser un valor más eficaz como El que los otros valores de SF empleados durante un proce- dimiento de CCA, y cuyo propósito fue establecer una mayor y mejor evaluación hacia la marca con que se le apareó. La comparación entre las medias otorgadas en la medida pre (antes del apareamiento) y los registros post (después del apareamiento) hace evidente las cualidades de este criterio para la elección de un El.

Los resultados de este estudio se suman a los aportes de Cruz, 2008 y Hens (2000), en la medida que dan soporte a otro atributo físico del atractivo como un estímulo biológicamente relevante desde el contexto de las formulaciones evolucionistas.

La investigación que aquí se presenta, adicionalmente, provee otra evidencia empírica que confirman la predilección observada en investigaciones previas, en donde se hace patente la mayor preferencia de los seres humanos por la alta simetría (Rhodes et al., 1998; Rentschler, et al., 1999; Jones y DeBruine, 2007) y que dicha preferencia es el producto de un mecanismo evolucionado para evaluar una pareja óptima (Fink y Penton-Voak, 2002).

Infortunadamente, los datos proporcionados en este estudio no revelan mayor evidencia sobre los efectos de valores aún más bajos de SF en la respuesta evaluativa (Noor y Evans, 2003; Príncipe y Langlois, 2011) y, en el mismo sentido, cuáles son los efectos de este estímulo en un procedimiento de CCA.

Con los datos aquí recolectados y con los resultados de Cruz (2008) se puede concluir que la Psicología Evolucionista puede proveer de una prometedora heurísitica que permite identificar ( $\mathrm{y}$, en consecuencia, predecir con una alta probabilidad de éxito) cuáles son aquellos estímulos biológicamente significativos que pueden ser utilizados en procedimientos de CCA en investigaciones de laboratorio, como también en el diseño de comunicaciones persuasivas en ambientes naturales (Saad, 2006).

Sin embargo, quedan varias preguntas para tener en cuenta en posteriores investigaciones, por ejemplo, ¿existen diferencias durante los procesos de adquisición y cambio de preferencias entre hombres y mujeres cuando es utilizado el "atrac- 
tivo" como variable independiente? Una pregunta que se deriva de las propias formulaciones evolucionistas, las cuales sostienen que la historia natural de cada uno de los sexos condujo a diferencias que fueron originadas en los problemas que cada uno de ellos debió enfrentar y que, por citar un caso, hoy observamos en aspectos tan comunes como la importancia que se le otorga a los rasgos físicos de los miembros del otro sexo (Buss, 2013). Una investigación que resulta especialmente relevante si se considera que machos y hembras difieren en su capacidad para distinguir asimetrías (Portales, Gil y Beltrami, 2009).

Otro problema de investigación que surge desde la lógica del diseño de comunicaciones persuasivas está relacionado con las prioridades de tratamiento que la audiencia otorga a las diferentes dimensiones del atractivo físico, por ejemplo, ¿predomina el procesamiento y valencia provocada hacia la SF sobre otros atributos físicos de atractivo? 0, por el contrario, ¿prima otra dimensión sobre el rostro? Aunque el estudio de Peters, Rhodes y Simmons (2007) parece sugerir la primacía de lo facial, es indudable que no hay estudios que confirmen o descarten este tema cuando se trata de la adquisición y cambio de actitudes.

Finalmente, es importante señalar que en solo el tema de SF se pueden derivar más preguntas de investigación (como por ejemplo, ¿cuáles asimetrías resultan más claves o de mayor incidencia en la evaluación de atractivo?), como también se pueden plantear cuestiones a partir de la tecnología empleada durante el la elaboración y presentación de los rostros (por ejemplo, ¿cómo incide la exposición de los estímulos cuando estos son en 3D o están en movimiento?).

\section{Referencias}

Aharon, I., Etcoff, N., Ariely, D., Chabris, C. F., O’Connor, E. \& Breiter, H. C. (2001). Beautiful faces have variable reward value: FMRI and behavioral evidence. Neuron, 32, 537-551.

Ajzen, I. (2001). Nature and operation of attitudes. Annual Review of Psychology, 52, 27-58.
Ajzen, I. \& Fishbein, M. (2005). The influence of attitudes on behavior. D. Albarracín, B. T. Johnson, \& M. P. Zanna (eds.). The handbook of attitudes, 173-221. Mahwah, N.J: Erlbaum.

Baker, M. J. \& Churchill, G. A. (1977). The impact of physically attractive models on advertising evaluations. Journal of Marketing Research, 14, 538-555.

Baudouin, J. Y. \& Tiberghien, G. (2004). Symmetry, averageness, and feature size in the facial attractiveness of women. Acta Psychologica, 117, 313-332.

Benjamin, L. T. (2004). Science for sale: Psychology's earliest adventures in American advertising. J. D. Williams, W. N. Lee, \& C. P. Haugtvedt (eds.), Diversity in advertising: Broadening the scope of research directions, 22-39. Mahwah, N. J.: Lawrence Erlbaum.

Bennett, R. \& Eckman, J. (1973). Attitudes toward aging: A critical examination of recent literature and implication for future research. C. Eisdorfer y M. P.Lawton (eds.). The psychology of adult development and aging, 96-115. Washington, D. C.: American Psychological Association.

Berridge, K. C. (2001). Reward learning: Reinforcement, incentives and expectations. The Psychology of learning and motivation, 40, 223-278.

Bower, A. B. (2001). Highly attractive models in advertising and the women who loathe them: The implications of negative affect for spokesperson effectiveness. Journal of $\mathrm{Ad}$ vertising, 30, 51-63.

Brewer, M. B. \& Lui, L. (1989). The primacy of age and sex in the structure of person categories. Social Cognition, 7(3), 262-272.

Briñol, P., de la Corte, L. y Becerra, A. (2001). Qué es persuasión. Madrid: Biblioteca Nueva

Briñol, P. \& Petty, R. E. (2012). The history of attitudes and persuasion research. A. Kruglanski 
\& W. Stroebe (Eds.). Handbook of the history of social psychology, 285-320. New York: Psychology Press.

Brownlow, S. (1992). Seeing is believing: Facial appearance, credibility, and attitude change. Journal of Nonverbal Behavior, 16(2), 101-115.

Buss, D. M. (2007). The evolution of human mating. Acta Psychologica Sinica, 39, 502-512.

Buss, D. M. (2013). Evolutionary Psychology: The new science of the mind (4th ed.). Boston: Pearson.

Cacioppo, J. T., Berntson, G. C. \& Decety, J. (2010). Social neuroscience and its relationship to social psychology. Social Cognition, 28(6), 675-685.

Cárdenas, R. A. \& Harris, L. J. (2006). Symmetrical decorations enhance the attractiveness of faces and abstract designs. Evolution and Human Behavior, 27, 1-18.

Cetinkaya, H., Dural, S. \& Gulbetekin, E. (2010). Testing the concealed ovulation hyphotesis in the framework of facial symmetry fluctuations moderated by menstrual cycle in women. Turkish Journal of Psychology, 25(66), 17-20.

Chaiken, S. (1979). Communicator physical attractiveness and persuasion. Journal of Personality and Social Psychology, 37, 1387-1397.

Colarelli, S. M. \& Dettman, J. R. (2003). Intuitive evolutionary perspectives in marketing practices. Psychology \& Marketing, 20(9), 837-865.

Crano, W. D. \& Prislin, R. (2006). Attitudes and persuasion. Annual Review of Psychology 57, 345-374.

Creusen, M. E., Veryzer, R. W. \& Schoormans, J. P. (2010). Product value importance and consumer preference for visual complexity and symmetry. European Journal of Marketing, 49(9-10), 1437-1452.
Cruz. J. E. (2008). Condicionamiento evaluador de preferencias en el consumidor a partir del atractivo físico. Revista Latinoamericana de Psicología, 40(2), 345-359.

Cuervo, J. J. (2000). El estudio de la asimetría fluctuante. Temas actuales en etología. Etologuía, 18, 27-60.

Dion, K., Berscheid, E. \& Walster, E. (1972). What is beautiful is good. Journal of Personality and Social Psychology, 24, 285-290.

Durante, K. M. \& Li, N. P. (2009). Oestradiol level and opportunistic mating in women. Biology letters, 5, 179-182.

Eagly, A. H., Ashmore, R. D., Makhijani, M. G. \& Longo, L. C. (1991). What is beautiful is good, but...: A meta-analytic review of research on the physical attractiveness stereotype. Psychological Bulletin, 110(1), 109-128.

Ellis, L. \& He, P. (2011). Race and advertising: Ethnocentrism or "real" differences in physical attractiveness? Indirect evidence from China, Malaysia, and the United States. The Mankind Quarterly, 51(4), 471-489.

Etcoff, N. (1999). Survival of the Prettiest. New York: Doubleday.

Fink, B. \& Penton-Voak, I. (2002). Evolutionary psychology of facial attractiveness. American Psychological Society, 11(5), 154-158.

Fink, B., Neave, N., Manning, J. T. \& Grammer, K. (2005). Facial symmetry and the big five personality factors. Personality and Individual Differences, 39, 523-529.

Froufe, M. y Sierra, B. (1998). Condicionamiento clásico de preferencias implicaciones para la publicidad. Revista de Psicología General y Aplicada, 51, 85-104.

Gangestad, S. W. \& Scheyd, G. J. (2005). The evolution of human physical attractiveness. Annual Review of Anthropology, 34, 523-548. 
Gould, S. J. (1979). Mickey Mouse meets Konrad Lorenz. Natural History, 88, 30-36.

Graham, J. H., Raz, S., Hel-Or, H. \& Nevo, E. (2010). Fluctuating asymmetry: Methods, theory, and applications. A review. Symmetry, 2, 466-540.

Grammer, K. \& Thornhill, R. (1994). Human (Homo Sapiens) facial attractiveness and sexual selection: The role of symmetry and averageness. Journal of Comparative Psychology, 108, 233-242.

Grammer, K., Fink, B., Moller, A. P. \& Thornhill, R. (2003). Darwinian aesthetics: Sexual selection and the biology of beauty. Biological Reviews, 78, 385-407.

Henss, R. (2000). Waist-to-hip ratio and female attractiveness. Evidence from photographic stimuli and methodological considerations. Personality and Individual Differences, 28(3), 501-513.

Horcajo, J., Briñol, P. \& Petty, R. E. (2010). Consumer persuasion: Indirect change and implicit balance. Psychology and Marketing, 27, 938-963.

Hovland, C. I., Janis, I. L. \& Kelley, J. J. (1953). Communication and persuasion: Psychological studies of opinion change. New Haven: Yale University Press.

Hughes, D. G. (1967). Selecting scales to measure attitude change. Journal of Marketing Research, 4(1), 85-87.

Hume, D. K. \& Montgomery, R. (2001). Facial attractiveness signals different aspects of "quality" in women and men. Evolution Human Behaviour, 22, 93-112.

Jasienska, G., Ziomkiewicz, A., Ellison, P. T., Lipson, S. F. \& Thune, I. (2006). Symmetrical women have higher potential fertility. Evolution and Human Behavior, 27, 390-400.

Jasienska, G., Ziomkiewicz, A., Ellison, P. T., Lipson, S. F. \& Thune, I. (2004). Large breasts and narrow-waists indicate high reproductive potential in women. Proceedings of the Royal Society of London B, 271, 1213-1217.

Jones, B. C. \& DeBruine, L. M. (2007). The role of symmetry in attraction to average faces. Perception \& Psychophysics, 69(8), 1273-1277.

Jones, B. C., Little, A. C., Penton-Voak, I. S., Tiddeman, B. P., Burt, D. M. \& Perrett, D. I. (2001). Facial symmetry and judgements of apparent health support for a "good genes" explanation of the attractiveness-symmetry relationship. Evolution and Human Behavior, 22, 417-429.

Joseph, W. B. (1982). The credibility of physically attractive communicators: A review. Journal of Advertising, 11(3), 15-24.

Kandel, E. R., Abrams, T., Bernier, L., Carew, T. J., Hawkins, R. D. \& Schwartz, J. H. (1983). Classical conditioning and sensitization share aspects of the same molecular cascade in aplysia. Cold spring harbor symposia quantitative biology, 48, 821-830.

Langlois, J. H., Kalakanis, L., Rubenstein, A. J., Larson, A., HaUam, M. \& Smoot, M. (2000). Maxims or myths of beauty? A meta-analytic and theoretical review. Psychological Bulletin, 126(3), 390-423.

Lipson, S. F. \& Ellison, P. T. (1996). Comparison of salivary steroid profiles in naturally occurring conception and non-conception cycles. $\mathrm{Hu}$ man Reproduction, 11, 2090-2096.

Nisbett, R. E. \& Wilson, T. D. (1977). The halo effect: Evidence for unconscious alteration of judgments. Journal of Personality and Social Psychology, 35(4), 250-256.

Noor, F. \& Evans, D.C. (2003). The effect of facial symmetry on perceptions of personality and attractiveness. Journal of Research in Personality, 37, 339-347.

Olson, M. A. \& Fazio, R. H. (2001). Implicit attitude formation through classical conditioning. Psychological Science, 12, 413-417. 
Olson, J. M., Vernon, P. A., Harris, J. A. \& Jang, K. L. (2001). The heritability of attitudes: A study of twins. Journal of Personality and Social Psychology, 80, 845-860.

Osgood, C. E., Suci, G. J. \& Tannenbaum, P. H. (1957). The measurement of meaning. Urbana, Illinois: University of Illinois Press.

Peters, M., Rhodes, G. \& Simmons, L. W. (2007). Contributions of the face and body to overall attractiveness. Animal Behaviour, 73, 937942.

Pornpitakpan, C. (2012). A critical review of classical conditioning effects on consumer behavior. Australasian Marketing Journal, 20, 282-296.

Portales, K., Gil, C. y Beltrami, M. (2009). Influencia de la simetría facial en la elección de pareja. Revista de Psicología, 18(2), 147-160.

Principe, C. P. \& Langlois, J. H. (2011). Faces differing in attractiveness elicit corresponding affective responses. Cognition and Emotion, $25,140-148$.

Rentschler, I., Jüttner, M., Unzicker, A. \& Landis, T. (1999). Innate and learned components of human visual preference. Current Biology, 9, 665-671.

Rescorla, R. A. (1988). Pavlovian conditioning: It's not what you think it is. American Psychologist, 43, 151-160.

Rhodes, G. (2006). The evolutionary psychology of facial beauty. Annual Review of Psychology, $57,199-226$.

Rhodes, G., Proffitt, F., Grady, J. \& Sumich, A. (1998). Facial symmetry and the perception of beauty. Psychonomic Bulletin \& Review, 5, 659-669.

Saad, G. (2004). Applying evolutionary psychology in understanding the representation of women in advertisements. Psychology \& Marketing, 21, 593-612.
Saad, G. (2006) Applying evolutionary psychology in understanding the Darwinian roots of consumption phenomena. Managerial and Decision Economics, 27, 189-201.

Schachtman, T. R. \& Reilly, S. (2011). Thigs you always wanted to know about conditioning but were afraid to ask. T. R. Shachtman \& Reilly (eds.). Associative learning and conditioning theory. Human and non-human applications, 481-506. Oxford: Oxford University Press.

Schachtman, T. R., Walker, J. \& Fowler, S. (2011). Effects of conditioning advertising. ET. R. Shachtman \& Reilly (eds.). Associative learning and conditioning theory. Human and non-human applications, 481-506. Oxford: Oxford University Press.

Shepherd, K. \& Bar, M. (2011). Preference for symmetry: Only on mars? Perception, 40, 12541256.

Schoenfeld, W. N. (1995). Encore. "reinforcement" in behavior theory. The Behavior Analyst, 18(1), 173-185.

Staats, A. W. \& Staats, C. (1958). Attitudes established by classical conditioning. Journal of Abnormal and Social Psychology, 67, 159-167.

Tesser, A. (1993). The importance of heritability in psychological research: The case of attitudes. Psychological Review, 100, 129-142.

Trampe, D., Stapel, D. A., Siero, F. W. \& Mulder, H. (2010). Beauty as a tool: The effect of model attractiveness, product relevance, and elaboration likelihood on advertising effectiveness. Psychology \& Marketing, 27(12), 1101-1121.

Van Valen, M. L. \& Macre, C. N. (2004). Is beautiful always good? Implicit benefits of facial attractiveness. Social Cognition, 22, 637-649.

Vyncke, P. (2011). Cue management: using fitness cues to enhance advertising effectiveness. G. Saad (ed.). Evolutionary Psychology in the 
business sciences, 257-287. Berlin: SpringerVerlag.

Wade. J. (2010). The relationships between symmetry and attractiveness and mating relevant decisions and behavior: A review. Symmetry, 2, 1081-1098.

Walster, E. \& Walster, G. W. (1963). Effect of expecting to be liked on choice of associates. Journal of Abnormal and Social Psychology, 67, 402-404.
Windholz, G. (1989). The discovery of the principles of reinforcement, extinction, generalization, and differentiation of conditional reflexes in Pavlov's laboratories. Pavlovian Journal of Biological Science, 24(2), 35-42.

Zebrowitz, L. A. (1997). Reading Faces: Window to the Soul? Boulder, CO, USA: Westview Press.

Zebrowitz, L. A. \& Montepare, J. M. (2008). Social psychological face perception: Why appearance matters. Social and Personality Psychology Compass, 2, 1497-1517. 